\title{
Innovations
}

\section{Cameos of Compassion: Exploring compassionate care using secondary analysis of digital patients' stories}

\section{Authors}

Bebhinn Dillane', Ivanka Ezhova², Sara Ryan³, Stephen $\mathrm{Tee}^{4}$, Anne Marie Rafferty ${ }^{\star}$

\section{Abstract}

Patient stories have been identified as a powerful tool to improve quality of care. Healthtalk.org is a digital resource presenting patients' experiences of illness and healthcare. Data have been generated from narrative interviews conducted by experienced researchers, based at the Health Experiences Research Group, University of Oxford. Our project explored the potential use of secondary analysis of digital sources as a methodological innovation to develop as a tool for teaching compassion to nursing students. We found that secondary analysis of narrative interviews provides a powerful resource for identifying positive and negative patient experiences for learning and teaching. These can be designed into a digital toolkit and used to develop students' reflexivity in relation to the values and behaviours associated with compassionate care and practice.

\section{Keywords}

nursing care, nursing education, $6 C^{\prime} s$, qualitative data, patient stories, secondary analysis, methodological innovation

\section{Introduction}

According to the Department of Health' and Kaufman et al., ${ }^{2}$ an educational experience that encourages compassion in practice and delivers a well-balanced programme compatible with the $6 \mathrm{C}^{\prime} \mathrm{s}$ - care, compassion, competence, communication, courage and commitment is a vital part of professional formation and fundamental entitlement of the nursing student experience. Findings in the Quality with Compassion Report, provided by the Willis Commission ${ }^{3}$ encourages students to become partners in their education as they are the future of nursing and the next generation of practitioners

Compassion in Practice, as proposed by the Chief Nursing Officer for England, ${ }^{4}$ saw the implementation of the $6 C^{\prime}$ 's initiative which represents the core values underpinning good
'Centre for Neuroscience and Trauma, Blizard Institute, London, United Kingdom

2Florence Nightingale Faculty of Nursing and Midwifery and Palliative Care, King's College London, United Kingdom

${ }^{3}$ Nuffield Department of Primary Care Health Sciences, University of Oxford, United Kingdom

${ }^{4}$ Faculty of Health and Social Sciences, Bournemouth University, United Kingdom

*Email: anne_marie.rafferty@kcl.ac.uk

\section{Cite this article as:}

Dillane B, Ezhova I, Ryan S, Tee S, Rafferty AM. Cameos of Compassion: Exploring compassionate care using secondary analysis of digital patients' stories. Innovations in Global Health Professions Education. 2018:3. https://dx.doi.org/10.20421/ ighpe2018.03

This is an open access article distributed under the terms of the Creative Commons Attribution license CC BY 4.0, which permits unrestricted use, distribution and reproduction in any medium, provided the original work is properly cited. 
care. These six fundamental nursing values aim to improve the standard of care received by patients and ensure staff are supported in the emotional labour of caring.

Similar projects had already been implemented in the United States. Transforming Care at the Bedside (TCAB), which is an American national programme of the Robert Wood Johnson Foundation (RWJF), ${ }^{5}$ engaged both frontline nursing and medical staff in improving patient outcomes and nurses' work environments. These focussed on providing more reliable, efficient and patient focused services, and support for more effective and adequate teamwork. ${ }^{6-8}$ TCAB started in 2003 in partnership with the Institute for Healthcare Improvement $(\mathrm{IHI})^{9}$ as a pilot study, including 13 hospitals where the initial evaluation of staff engagement and performance was positive. The project was expanded in 2007, into a 67-hospital collaborative, overseen by the American Organization of Nurse Executives (AONE)6 and was positively evaluated in terms of engaging staff in the workplace and innovations tested. ${ }^{6,9-12}$

In the United Kingdom, the $6 \mathrm{C}^{\prime}$ 's have proven to be a powerful rhetorical device around which the profession could rally and build a strong and coherent sense of identity. They emerged however, at a particular moment in time, namely the aftermath of the Francis Inquiry into the scandals of care in Mid Staffordshire NHS Foundation Trust (Report of the Mid Staffordshire NHS Foundation Trust Public Inquiry) ${ }^{13}$ when the reputation of the profession had been tarnished. The appointment of a new Chief Nurse carried the expectation that a moral and spiritual renewal of the profession was needed. As discussed by Jones ${ }^{14}$ it is important for students to be recruited to universities with the correct values even though there is little evidence on how best to do this in terms of the impact on educational or clinical outcomes. Support is then needed to nurture and encourage student nurses and their values. Teaching modules based around, and incorporating the concepts of compassion and care have emerged within universities. Much of this though may be making explicit what has often been tacit or taken for granted in the curriculum. ${ }^{15-17}$

As stated in the Willis Commission on preregistration nurse education in England ${ }^{3}$ the involvement of patients and the public in pre- registration nurse education provides a significant contribution to the quality of the educational experience for students and must be implemented using the Nursing Midwifery Council for the UK Standards. ${ }^{18}$ Similar to the way we have learned from hearing the personal stories of the Mid Staffordshire NHS Foundation Trust victims of poor care to improve care, ${ }^{13}$ patient stories told on e-health resources such as HealthTalk.org can potentially influence nursing practice and education. The use of patient stories is favoured in much of the literature as it generates discussion, reflection and helps students understand the complex process of delivering compassionate care alongside transforming how students view themselves and their patients. ${ }^{19-21}$

The value of patient stories is gaining increasing support within the international literature. During the past decades, significant numbers of healthcare professionals worldwide have raised questions about how giving voice to patients and caregivers improves the experience of care, safety and quality, as well as the learning environments for students and trainees. ${ }^{22-24}$ Thomason, Toman and Potter ${ }^{25}$ reported that patient stories add physical essence to sensitive concepts like compassion, collaboration, dignity, respect, pain and information sharing.

Stories put faces to statistics and patient experience scores. Storytelling is a valuable tool for engaging patients and those close to them as a mechanism to develop higher-level empathy skills by care providers. Recently, the growing use of the Internet has popularised and made more accessible patient experiences. According to Ziebland and Wyke, ${ }^{26}$ there are more than 34 online free access resources that provide health information and support across the United Kingdom. Health policymakers have also started to recognise the potential of the Internet as a valuable source of patients' views and experiences. For instance, The Operating Framework for the NHS in England 2011/1227 highlights the importance of patients' perspectives on how the system operates (or not); the NHS Future Forum recommendations to Government - second phase $2012^{28}$ talks about transparency, public voice and the digital revolution.

The internet could also improve nursing education and nursing students' experiences. Christianson ${ }^{29}$ concluded that the use of digital material is most effective in student education as it allows students to explore their own thoughts and beliefs regarding 
practice, their reflexivity. They can change personal perspectives in how the students view themselves as care givers and their patients and furthermore how they prioritise their main concerns and those of their patients. It is vital students have this selfawareness when in practice and through the use of a professional development tool, this can be promoted alongside active learning, as highlighted by Moon and Fowler. ${ }^{30}$ Jones and Ellis ${ }^{14}$ explored the role universities now have in establishing that prospective students possess the necessary nursing values and also to confirm they can demonstrate compassion in care during the student recruitment process. Such recruitment strategies have been recommended by the Francis Inquiry ${ }^{13}$ and the Care Quality Commission Report ${ }^{3}$ to ensure recruits have potential to be compassionate and competent qualified nurses.

Such studies and reports support the use of patient stories in education and indicate that there are many benefits potentially to be gained from listening to storytellers. This article reports on a pilot study designed to explore the potential role that secondary analysis of personal stories available on archived material and websites such as HealthTalk.org could play in nursing education by demonstrating how service user stories related to the $6 C^{\prime}$ 's might inform student learning in this area.

\section{Process}

The study used secondary analysis of qualitative data of patient experiences contained within a data archive of patient and carer experience of health care. ${ }^{31}$ The findings were obtained from the Health Experience Research Group (HERG) interview archive. This archive contains data, collected nationally by experienced social scientists who work within the HERG, using qualitative interviews. The archive is private, only accessed with authorisation by the HERG and currently composed of over 75 collections, related to different health conditions (e.g. experience of pregnancy, terminal illness). Each set is formed by 35-50 interviews, digitally audio or video recorded, transcribed verbatim and copyrighted to the University of Oxford for non-commercial purposes, including publications and secondary analysis.
HERG's research aims to find out relevant and specific information about patients and relatives' experiences of healthcare across the United Kingdom. The research methodology combines open-ended and semi-structured questions, inciting narrative and/or more focused responses about treatment decisions, information, support and communication with NHS professionals at all levels. The goal is to provide instructive data about how health services and communication could be improved. The use of diverse samples have generated a broad spectrum of patient experiences, varying from what could be considered as "typical" to more unusual experiences. Analysis of the data have been published in peer-reviewed journals over the past ten years. ${ }^{26,32,33} \mathrm{~A}$ summary of research findings is also available online via the HERG website Healthtalk.org. The website is a public source of patient experience approved by the NHS Information Standard and cited in the NHS Evidence Process and Methods Manual. ${ }^{26}$

This study utilised secondary analysis of HERG's archive interview collections using the following key words: workforce, 6C's, nursing, nursing education, care, compassion, patient stories, and relative. The initial search included a large number of physical and mental conditions across all ages, treated mainly in acute care settings. In healthcare, secondary analysis involves the use of existing data, collected for a particular purpose, distinct from the original study. This may relate to a new research topic or research question relevant to the original study. ${ }^{34}$

The search terms cited above were applied to a random sample of excerpts by a member of the HERG. Relevant data related to patients' experiences of nursing care and communication were extracted from these transcripts. The data were linked to the $6 C^{\prime}$ 's in order to identify material of potential use for educational reflections and encourage the use of $6 C^{\prime}$ 's in nursing and education at undergraduate and postgraduate level by one of us (BD) making the undergraduate research fellow aware of patients and relatives' perspectives regarding communication and healthcare experience. After being linked to the $6 C^{\prime}$ 's, data were analysed and discussed, using the critical reflection framework, based on the critical incident technique developed by Flanagan. ${ }^{35}$ 
Flanagan's conceptualisation enables researchers to, "reflect-for-action", i.e. describes actions that could be undertaken and/or improved in future experiences. The technique helps analyse what works well or poorly, and why; formulate problems in general terms, so they could apply findings to a large range of issues; identify contributing factors to the failure or success of specific situations; and develop new research methods. The critical reflection framework is a guided process to analyse and increase the potential for positive outcomes of positive or negative critical incidents. The framework is specifically useful in development and enquiry orientated programmes, related to learning, teaching and in synchronisation with the participants' experiences and needs, ${ }^{36}$ Critical reflection can be used in different ways to create individual and/or group narratives, based on the recall of an experience. It can focus on a particular event or situation, or treat different experiences and perspectives, related to specific problems and issues.

This is the first study we know of where secondary analysis of digital qualitative data of patient experiences has been linked to the $6 C^{\prime}$ 's and explored for its potential transformation into a toolkit to facilitate reflexive compassion in care. We explore the potential of this as a trigger for innovating nurse education.

\section{Results: Critical reflections on patients' stories}

The transcripts highlighted many aspects of positive and negative nursing care received by patients and their relatives during their hospital stay. Both types of experiences have been categorised under the headings of the $6 C^{\prime}$ 's as a thematic framework to illustrate how the tool could be beneficial in eliciting patient stories accessed from resources such as HealthTalk.org in the future of nursing education.

\section{Care}

Key themes regarding both positive and negative experiences of care were identified.

Positive experiences include: introductions being made between patient and service providers and agreeing on preferred names; attention to basic care needs, nurses engaging with and talking to patients and 'checking in' with them; emotional support for the patients and their family; demonstration of interest in the patient as an individual and not as a hospital number or illness; being aware of professional boundaries; discussions with the patient about improvement in their care experience, e.g.

[They did...] things that matter to you. I was thoroughly washed every day. It made me feel human having my hair washed and feeling clean. Some of the nurses went out of their way to help.

In contrast, a range of transcripts identified major concerns regarding nursing care. Themes illustrating poor nursing care include: nurses being 'rough' when carrying out personal hygiene tasks; unacceptable hygiene standards (with reports of nurses not washing their hands or using gels between patient visits), frequent criticism regarding nurses' attitudes and professional behaviours.

\section{Compassion}

On the one hand, treating patients with respect and dignity was recognised by patients as being a vital aspect of positive care experiences. The value of care, taking the time to get to know patients and being able to support them was viewed as a compassionate value by the service users. It was evident that building positive relationships is greatly appreciated by service users and their relatives and instils confidence in their nurse. Even when a patient is unconscious, relatives recognise that the nurse can still respect the patient by speaking to them and explaining what is going on around them.

The following example of a mental health patient demonstrates patients' admiration for the nurses for building open, communicative and friendly relationships, e.g.

The nurses I really like because they're interested in me as a person, not just as a patient. They all know I draw and they ask about it. They will all know my husband and they talk to him and we get along with them very well, and if I end up there I get a good hug, which is really nice.

On the other hand, another mental health patient reported an unnecessary increase in his medication, 
feelings of being ignored and labelled by the nursing team. The patient explained how he felt being judged, e.g.

Because you get judged on every word, you start to close up. The people you should open up to and that are there for you, and lots of people with schizophrenia will recognise this, you actually don't open up. I learnt to stop opening up to them because they were judging every word I said. And saying: you should increase your medication.

Some patients also described negative experiences, such as being excluded from their care; significant lack of support for patient and relatives; lack of understanding of the patient journey and illness; little respect concerning dignity; huge divergence between professionals and relatives' perspectives about care administration.

\section{Competence}

Highly trained, knowledgeable and competent nurses are readily identified by service users. The interviews demonstrated strongly that if the patients consider the nurse as competent and professional, their confidence and experience of healthcare increase immediately. Other key factors related to competence include: sharing relevant information, understanding and adapting to the patient's environment, being in possession of good judgement skills, being able to work as part of a multidisciplinary team and understanding the patient's situation through a combination of knowledge, professional experience and empathy. Negative factors include: lack of competence, skills, knowledge and confidence. Another issue the patients discussed was the transfer from intensive care unit to the ward where a large number of service users described ward nurses as less qualified and less able to provide care for their patients compared to intensive care unit nurses. Despite these observations, patients recognised that ward nurses could be underresourced and under-staffed. Patients are aware that these factors contribute to poor care experience, e.g.

Going to the ward wasn't pleasant. I think it was a real culture shock to be honest. [A relative explained regarding his wife's move from intensive care to the ward]. It was like going from a 5-star hotel to a very, very low level B \& B.
Carers expressed their frustration at the challenges of engaging them in care in a timely fashion. A worried and angry mother showed concern for her child as she needed to constantly run after staff. The need to 'chase' nurses was highlighted as a major issue surrounding care and the conflict this created when they were officially "on" and "off" duty, wrestling with a competing set of priorities, e.g.

Our child is also your responsibility and you cannot say to me that it is not [your] responsibility and that it is not [your] shift. It is your shift, it is all of your shifts and our child is your responsibility.

\section{Communication}

Patients raised concerns about the use of medical jargon by doctors and the need to repeatedly ask nurses for clarification and/or interpretations of what doctors were saying. The role of the nurse as an intermediary between the patient and medical staff was an important feature of communication. Thus, the capacity to be patient when communicating with patients and relatives was valued by participants.

Skill and dexterity in handling difficult areas of communication as well as being prepared to repeat and reinforce key messages were appreciated by patients and families. The following example demonstrates the degree to which nurses made themselves available to relatives and the impact on improving relatives' experience of healthcare, e.g.

They make you feel at ease and they explain that you can ask questions again and again and again. And it doesn't matter how many times you ask them, they won't mind... because they communicate with you, that's how they find out about their patient.

Nurses talking with unconscious patients as well as relatives was remarked upon and greatly appreciated by relatives as revealing great sensitivity, e.g.

They always used to sit and talk to you, even though you couldn't answer them. I used to go in there some days and the nurse would be talking to you. One of them was telling you about her son getting married. 
Positive communication also seemed to help "normalise" what was an alienating experience for both relatives and patient.

Negative aspects of communication comprised: lack of correct or sufficient information, generated by medical and nursing staff, exclusion of the patients and their relatives from decisions. A mother of a new born with Congenital Heart Disease (CHD) describes feelings of exclusion regarding her son's care in intensive care unit, e.g.

It was very busy, very, very serious, several nurses taking care of [our son] and I just felt so not a part of it. It's a natural thing, you want to be taking care of your baby so in the end I just went out in tears.

On the negative side, factors such as poor nonverbal skills and the poor communication of bad news also featured in patient stories.

\section{Courage}

Overall, little was mentioned about the value of courage. Some patients and relatives did notice however, some nurses being very much involved in case meetings, joining doctors on ward rounds and questioning doctors regarding aspects of patient care. Being able to speak out about hard issues with empathy and competency was also recognised, alongside the need to manage demanding situations and scenarios with patients, members of the public and colleagues, e.g.

I think it made so much difference to me to be looked after by their method which is a very strong leadership and a comprehensive team approach with everyone involved. All the nurses were involved in team meetings and it really showed, the nurses were brilliant, very well trained.

The courage to take responsibility was another key theme discussed in the patient's stories, e.g.

It is about building up that trust with a family and encouraging them to believe in what you are saying and that you are being honest. If something does go wrong that is your fault, you must tell the family, "look we did this and it has happened.
Inadequate internal communications as well as handover were the main target in patients stories of poor care experiences. Nurses who didn't seek more information or clarification were perceived as being under engaged and their competence was questioned. The mother of another little boy with Congenital Heart Disease (CHD) felt as though the handover from intensive care unit to the ward was inadequate and undermined parents' confidence in staff, e.g.

The day before his operation the nurse didn't even know that he'd had surgery and what he was in for. It just made us feel a bit, "This isn't very good. They obviously have a lot of changeovers and we just found that information wasn't getting passed on. And we found ourselves repeating ourselves a lot.

\section{Commitment}

Aspects of care that impacted patient experiences positively included: observing nurses enjoying their work; nurses remembering their patients and making them feel like a person rather than a number or illness; "being there" and supporting patients and their relatives; accessibility and availability of the nurses to patients and relatives. A good example of commitment was when formally patients were inspired by the quality of nursing engagement in care, restoring his confidence in the system having previously been on the receiving end of poor care, e.g.

There isn't anything they can't do. They are powerful people and when you think of the nursing I don't know how they do some of the tasks, tremendous really. It's restored my faith in hospital care.

Nurses displaying high calibre knowledge, skills and commitment to high quality care was praised by patients and families.

Patients nonetheless experienced feelings of being ignored and/or abandoned. Unacceptable attitudes and unmet patient requests being told ("it's not my shift") were also described. One mother raised a concern about the care of her son's cannula. She explained how the site had been bandaged and neglected by staff over the course of the week, e.g. 
When he vomited... the milk would go on that bandage and it would soak through, seep through more and more and get soggy and after three days the thing starts to stink.

\section{Discussion}

This exploratory pilot study demonstrates the use of secondary analysis from a data archive on patients' and relatives' experiences of healthcare using the $6 \mathrm{C}^{\prime}$ 's as a framework for analysis. We identified ways in which the $6 C^{\prime}$ 's could be identified within patient stories of healthcare experience using online data resources, such as Healthtalk.org. Both the positive and negative stories told by service users illustrate the impact that nurses' work, the culture within which healthcare work is embedded, values and attitudes can have on the patient's and relatives' experiences in hospital.

The benefits of using patients' stories to improve healthcare experience, education and workforce have been identified in the international literature. ${ }^{24,29,37}$ Johnson $^{22}$ developed four core concepts of patient- and family-centred care, i.e. dignity and respect, information sharing, participation and collaboration. Thomason, Toman and Potter ${ }^{25}$ reported that patient stories add physical essence to invisible concepts like pain, compassion, anguish, and collaboration, emphasising that stories put faces to statistics, and remind health care professionals why they chose to do what they do in the first place. Understanding the illness process from the patient's perspective brings caregivers, and nurses in particular, important and valuable feedback on how to engage in best practices that create healing contexts for patients and their families. It also recognised patients as unique individuals increases the potential for trust and openness, and provides a foundation for giving them voice. ${ }^{38,39}$

This study suggests that the $6 C^{\prime}$ 's has some resonance with patient and carer accounts of care and that student learning might be further enhanced with the use of 6C's as a rubric for reflection on the quality process. A new nursing strategy has been launched in England but this does not invalidate the currency that the $6 C^{\prime}$ 's provides as a rhetorical device with which to think about quality of care.
The use of digital stories by service users within education can be most effective when used as an opportunity for students to reflect upon and re-evaluate their experience from a different perspective, and also for them to gain a new insight into their own practice. ${ }^{39} \mathrm{~A}$ lot can be learned from the attitudes of nurses expressed by storytellers in the data used for this study.

Given that accounts from patients and families were opportunistic, insofar as the purpose of the data collection was not to focus on nursing specifically but to explore the potential use of health care experiences of patients and carers for teaching and learning purposes more generally, it is significant that references to nursing care were made. As the first study, as far as we are aware, to explore the use of this data archive using secondary analysis it appears that this has some mileage in pinpointing what is important to patients and their relatives and carers about their experience of care. Many of the criticisms of care seemed to revolve around poor communication marked either by its absence - patients being ignored or having difficulty gaining the attention of nurses or engaging nurses in their care - having to "chase". Other issues included values, attitudes and forms of communication. The reference to "not my shift" does reflect an attitude of bounded work but there are ways of communicating the same information in a way that makes the person feel valued.

Negative cases could be used as material to stimulate learning for students' role playing and rewriting the script by reframing it as something more positive, e.g. "I'm no longer on duty but I shall find someone to help". Exercises such as these could highlight to students the importance of their beliefs and responsibilities in practice and allow them to become more self-aware and engage in reflection to improve their care. ${ }^{21}$

Group work with case based learning has also been greatly encouraged by students as they believe it enhances their learning experiences as well as educators who see it as a chance to get students to engage actively in their learning and motivates them to learn and question topics further, as also explored by Thistlethwaite, et al. ${ }^{40}$ Consistent with this, Terry's $\mathrm{s}^{41}$ study supports this method of learning as students found the use of digital 
patient stories an effective educational tool allowing discussion online and self-reflection and found that it encouraged even the quieter students to speak up. With compassionate care being encouraged throughout nursing practice and education, the combination of digital patient stories and the $6 C^{\prime}$ 's as a framework could provide the basis for developing an on line teaching and teaching tool to enable reflexivity in care for students. Engaging with patient stories is an essential key in learning as highlighted by Gidman ${ }^{19}$ who reported that students value time spent listening to patients.

We have tested the feasibility of identifying instances of nursing within the HERG digital archive and in doing so, have extracted those from the context in which they were generated. We have little contextual data on the embedded nature of nursing within broader accounts and this is a limitation of the study but the purpose of the pilot study was to explore the feasibility of identifying material useful for development into an on-line teaching and learning tool using digital patient stories as the source and interface. According to Aiken et al. ${ }^{42}$ identifying conditions supportive of compassionate practice could also provide useful data for patient and student engagement in co-design of the care environment. Our pilot study was designed to test the feasibility of the method of identifying episodes of nursing care within a digital archive and exploring their potential as a tool for reflective learning. The next step would be to develop the tool and test it by designing a collage of clips or cameos of care into a toolkit demonstrating different aspects of care and inviting students to reflect upon and provide feedback from the cameos and assess the implications for and from their own experience and practice.

\section{Conclusion}

This study adds to the current evidence of the use of digital patient stories and explores the feasibility of using these within nursing education using the $6 C^{\prime}$ 's as a framework for analysis, teaching and learning. The data illustrates how the fundamental values of care can be identified in service user experiences and potentially used to teach students about compassionate care. It is important that educational tools are available to encourage and nurture student values as compassionate care is a complex process especially within the pressurised environments of contemporary health care. The data could be designed into a toolkit and used to encourage discussion and reflection from student nurses, may help build resilience and improve care through self-transformation, awareness and provide critical support. High quality and compassionate behaviours are fundamental to the nursing profession.

Facilitating this within an educational framework has potential to embed such behaviours in practice. Training to be a nurse can be a stressful experience, especially in challenged care environments. Being able to support nursing students during a period of stress is essential and such educational tools hold the potential to build student resilience and provide service users with better and more positive care experiences.

Acknowledgements: We would like to thank King's College London Undergraduate Research Fellowship (KURF) Scheme for providing funding for Bebhinn Dillane.

Conflicts of interest: None.

Funding sources: King's College London Undergraduate Research Fellowship (KURF) Scheme.

\section{References}

1. Commissioning Board Chief Nursing Officer and DH Chief Nursing Adviser. Compassion in practice: nursing, midwifery and care staff: our vision and strategy [Internet]. London, UK: Department of Health, UK; 2012 Dec 4 [cited 2018 Sep 5]. Available from: https://www.england.nhs. uk/wp-content/uploads/2012/12/compassion-inpractice.pdf

2. Kaufman $\mathrm{G}$, Kondo $\mathrm{M}$, Knowles $\mathrm{C}$, Langford C, Leigh R, Lindsay $K$, et al. Nursing students' reflections on the Francis report. Nurs Stand. 2014 Sep 23;29(3):37-43. doi:10.7748/ns.29.3.37. e9102

3. Willis Commission on Nursing Education. Quality with compassion: the future of nursing education [Internet]. Report of the Willis Commission on Nursing Education, 2012: Royal College of Nursing on behalf of the independent Willis Commission 
on Nursing Education; 2012 [cited 2018 Sep 5]. Available from: https://www.macmillan.org.uk/ documents/newsletter/willis-commission-reportmacmail-dec2012.pdf

4. Cummings J, Bennett V. Compassion in practice: nursing, midwifery and care staff - our vision and strategy [Internet]. Leeds: NHS Commissioning Board; 2012 Dec 4 [cited 2018 Sep 6]. Available from: https://www.england.nhs.uk/wp-content/ uploads/2012/12/compassion-in-practice.pdf

5. Robert Wood Johnson Foundation, Institute for Healthcare Improvement. A new era in nursing: transforming care at the bedside [Internet]. 2006 Apr 10 [cited 2018 Sep 5]. Available from: https:// www.rwjforg/content/dam/files/legacy-files/ article-files/2/TCABBrochure041007.pdf

6. Needleman J, Pearson ML, Upenieks VV, Yee T, Wolstein J, Parkerton M. Engaging frontline staff in performance improvement: the American Organization of Nurse Executives implementation of Transforming Care at the Bedside Collaborative. Jt Comm J Qual Patient Saf. 2016 Feb;42(2):61-9. doi:10.1016/S1553-7250(16)42007-6

7. Dearmon V, Roussel L, Buckner EB, Mulekar M, Pomrenke B, Salas S, et al. Transforming Care at the Bedside (TCAB): enhancing direct care and value-added care. J Nurs Manag. 2013 May;21(4):668-78. doi:10.1111/j.13652834.2012.01412.x

8. Robert Wood Johnson Foundation [Internet]. Princeton, NJ: Robert Wood Johnson Foundation; c2001-2018. The transforming care at the bedside (TCAB) Toolkit; 2008 Jun [cited 2018 Sep 5]; [about 1 screen]. Available from: https:// www.rwjf.org/en/library/research/2008/06/thetransforming-care-at-the-bedside-tcab-toolkit. $\underline{\text { html }}$

9. Institute for Healthcare Improvement [Internet]. Boston, MA; Institute for Healthcare Improvement. Initiatives: transforming care at the bedside [cited 2018 Sep 5]; [about 3 screens]. Available from: http://www.ihi.org/engage/ initiatives/completed/TCAB/Pages/default.aspx

10. Parkerton PH, Needleman J, Pearson ML,
Upenieks VV, Soban LM, Yee T. Lessons from nursing leaders on implementing TCAB. Am J Nurs. 2009 Nov;109(11 Suppl):71-6. doi:10.1097/01. Naj.0000362030.08494.22

11. Yee T, Needleman J, Pearson M, Parkerton P. Nurse manager perceptions of the impact of process improvements by nurses. J Nurs Care Qual. 2011 Jul-Sep;26(3):226-35. doi:10.1097/ NCQ.0b013e318213a607

12. Pearson ML, Needleman J, Beckman R, Han B. Facilitating Nurses' Engagement in Hospital Quality Improvement: The New Jersey Hospital Association's Implementation of Transforming Care at the Bedside. J Healthc Qual. 2016 Nov/Dec;38(6):e64-e75. doi: 10.1097/ JHQ.0000000000000007

13. Report of the Mid Staffordshire NHS Foundation Trust Public Inquiry [Internet]. London, England: The Stationary Office; 2013 Feb 6 [cited 2018 Sep 5]. Available from: http://webarchive. nationalarchives.gov.uk/20150407084949/http:// www.midstaffspublicinquiry.com/sites/default/ files/report/Executive\%20summary.pdf

14. Jones $\mathrm{H}$, Ellis L. Putting the $6 \mathrm{Cs}$ at the heart of nurse education. Nurs Times [Internet]. 2014 2014/10/17 [cited 2018 Sep 4]; 110(37):12-4. Available from: https://www.nursingtimes.net/ Journals/2014/09/05/b/d/e/Putting-the-6Cs-atthe-heart-of-nurse-education-100914.pdf

15. Fry $\mathrm{H}$, Ketteridge $\mathrm{S}$, Marshall S. A handbook for teaching and learning in higher education: enhancing academic practice. 3rd ed. New York, NY: Routledge; 2009. 525 p.

16. Adamson E, Cook F, Crowther C, Dewar B, Donaldson JH, Gloag A, et al. Leadership in compassionate care: executive summaryenhancing patient care by promoting compassionate practice [Internet]. Edinburgh, Scotland: Edinburgh Napier University/NHS Lothian; 2012 [cited 2018 Sep 5]. Available from: http://researchrepository.napier.ac.uk/6207/7/ Compassionate Care Executive Report.pdf

17. Expert Panel on Global Nursing \& Health. Standards of practice for culturally competent 
nursing care [Internet]. Transcultural Nursing Society; 2010 Dec 30 [cited 2018 Sep 5].

Available from: https://tcns.org/wp-content/ uploads/2018/03/Standards of Practice for Culturally_Compt_Nsg_Care-Revised_.pdf

18. NMC: Nursing \& Midwifery Council [Internet]. London, UK; Nursing \& Midwifery Council; c2002. The Code for nurses and midwives: Read The Code online; 2008 Mar [cited 2018 Sep 5]; [about 15 screens]. Available from: https://www.nmc.org. uk/standards/code/read-the-code-online/

19. Gidman J. Listening to stories: valuing knowledge from patient experience. Nurse Educ Pract. 2013 May;13(3):192-196. doi:10.1016/j.nepr.2012.09.006

20. Pryce-Miller M, Vernel E. Developing compassion in pre-registration education. Nurs Times [Internet]. 2014 2014/10/17 [cited 2018 Sep 4]; 110(37):17-9. Available from: https://www. nursingtimes.net/roles/nurse-educators/ developing-compassion-in-pre-registrationeducation/5074552.article

21. McDrury J, Alterio M. Learning through storytelling in higher education: using reflection and experience to improve learning. London, UK: Kogan; 2003. 200 p.

22. Johnson BH. Promoting patient- and familycentered care through personal stories. Acad Med. 2016 Mar;91(3):297-300. doi:10.1097/ acm.0000000000001086

23. Johnson $B$, Abraham $M$, Conway J, Simmons L, Edgman-Levitan S, Sodomka $\mathrm{P}$, et al. Partnering with patients and families to design a patient- and family- centered health system: recommendations and promising practices [Internet]. Bethesda, MD: Institute for Family-Centered Care/Institute for Healthcare Improvement; 2008 April [cited 2018 Sep 6]. Available from: http://www.hqontario.ca/ Portals/O/modals/qi/en/processmap pdfs/ articles/partnering\%20with\%20patients\%20 and\%20families\%20to\%20design\%20a\%20 patient-\%20and\%20family-centered\%20health\%20 care\%20system.pdf

24. Tee S. Person-centred approaches in healthcare: a handbook for nurses and midwives. New York, NY: Open University Press; 2016. 292 p.

25. Thomason $\mathrm{M}$, Toman $\mathrm{M}$, Potter M. Giving a voice to patients and caregivers. N C Med J [Internet]. 2015 Jul-Aug [cited 2018 Sep 3]; 76(3):1657. Available from: https://doi.org/10.18043/ ncm.76.3.165

26. Ziebland S, Wyke S. Health and illness in a connected world: how might sharing experiences on the internet affect people's health? Milbank Q [Internet]. 2012 Jun 18 [cited 2018 Sep 3]; 90(2):219-49. Available from: https://www. ncbi.nlm.nih.gov/pmc/articles/PMC3460203/. doi:10.1111/j.1468-0009.2012.00662.x

27. DH/NHS Finance, Performance and Operations. The Operating Framework for the NHS in England 2011/12 [Internet]. London, UK: Department of Health; 2010 Dec 15 [cited 2018 Sep 6]. Available from: https://assets.publishing.service.gov. uk/government/uploads/system/uploads/ attachment_data/file/216187/dh_122736.pdf

28. NHS Future Forum. NHS Future Forum summary report: second phase overarching report. London, UK: Department of Health and Social Care; 2012 Jan 10 [cited 2018 Sep 6]. Available from: https:// assets.publishing.service.gov.uk/government/ uploads/system/uploads/attachment_data/ file/216422/dh_132085.pdf

29. Christiansen A. Storytelling and professional learning: a phenomenographic study of students' experience of patient digital stories in nurse education. Nurse Educ Today. 2011 Apr;31(3):28993. doi:10.1016/j.nedt.2010.10.006

30. Moon J, Fowler J. 'There is a story to be told...'; a framework for the conception of story in higher education and professional development. Nurse Educ Today. 2008 Feb;28(2):232-9. doi:10.1016/j. nedt.2007.05.001

31. Szabo V, Strang VR. Secondary analysis of qualitative data. ANS Adv Nurs Sci. 1997 Dec;20(2):66-74.

32. Ziebland S, Lavie-Ajayi M, Lucius-Hoene G. The role of the Internet for people with chronic 
pain: examples from the DIPEx International Project. Br J Pain [Internet]. 2015 Feb1 [cited 2018 Sep 3]; 9(1):62-4. Available from: https://www. ncbi.nlm.nih.gov/pmc/articles/PMC4616990/. doi:10.1177/2049463714555438

33. Ziebland S, Hunt K. Using secondary analysis of qualitative data of patient experiences of health care to inform health services research and policy. J Health Serv Res Policy. 2014 Jul;19(3):177182. doi:10.1177/1355819614524187

34. Hinds PS, Vogel RJ, Clarke-Steffen L. The possibilities and pitfalls of doing a secondary analysis of a qualitative data set. Qual Health Res. 1997 Aug 1;7(3):408-424. doi:10.1177/104973239700700306

35. Flanagan JC. The critical incident technique. Psychol Bull. 1954 Jul;51(4):327-58.

36. DiRanna K. Assessment-centered teaching: a reflective practice. Thousand Oaks, CA: Corwin Press; 2008. 205 p.

37. Stacey G, Hardy P. Challenging the shock of reality through digital storytelling. Nurse Educ Pract. 2011 Mar;11(2):159-64. doi:10.1016/j. nepr.2010.08.003

38. Schwind JK, Fredericks S, Metersky K, Porzuczek VG. What can be learned from patient stories about living with the chronicity of heart illness? A narrative inquiry. Contemp Nurse. 2016 AprJun;52(2-3):216-29. doi:10.1080/10376178.2015.108 9179

39. Jolly K, Weiss JA, Liehr P. Understanding adolescent voice as a guide for nursing practice and research. Issues Compr Pediatr Nurs. 2007 Jan-Jun;30(1-2):3-13. doi:10.1080/01460860701366518

40. Thistlethwaite JE, Davies D, Ekeocha S, Kidd JM, MacDougall C, Matthews $P$, et al. The effectiveness of case-based learning in health professional education. A BEME systematic review: BEME Guide No. 23. Med Teach. 2012;34(6):e421-44. doi:10.3109/014215 9x.2012.680939
41. Terry LM. Service user involvement in nurse education: a report on using online discussions with a service user to augment his digital story. Nurse Educ Today. 2012 Feb;32(2):161-6. doi:10.1016/j.nedt.2011.06.006

42. Aiken LH, Sloane DM, Bruyneel L, Van den Heede K, Griffiths P, Busse R, et al. Nurse staffing and education and hospital mortality in nine European countries: a retrospective observational study. Lancet [Internet]. 2014 May 24 [cited 2018 Sep 3]; 383(9931):1824-30. Available from: https://www. ncbi.nlm.nih.gov/pmc/articles/PMC4035380/. doi:10.1016/s0140-6736(13)62631-8 